\title{
Quaderni
}

QUADERNI Communication, technologies, pouvoir

83 | Hiver 2013-2014

Le public de la danse contemporaine

\section{Les représentations des publics dans le monde de la danse contemporaine}

Audience representations in the world of contemporary dance

\section{Daniel Urrutiaguer}

\section{(2) OpenEdition}

\section{Édition électronique}

URL : http://journals.openedition.org/quaderni/762

DOI : 10.4000/quaderni.762

ISSN : 2105-2956

\section{Éditeur}

Les éditions de la Maison des sciences de l'Homme

\section{Édition imprimée}

Date de publication : 5 janvier 2014

Pagination : 29-40

\section{Référence électronique}

Daniel Urrutiaguer, "Les représentations des publics dans le monde de la danse contemporaine », Quaderni [En ligne], 83 | Hiver 2013-2014, mis en ligne le 01 janvier 2016, consulté le 20 avril 2019.

URL : http://journals.openedition.org/quaderni/762 ; DOI : 10.4000/quaderni.762 


\section{$D$ ossier}

\section{Les représentations des publics dans le monde de la danse}

contemporaine

Longtemps considérée comme un parent pauvre des politiques culturelles, la danse contemporaine a bénéficié d'une reconnaissance institutionnelle par le ministère de la Culture et de la Communication sous les mandats de Jack Lang à la suite du doublement de son budget en 1982. L'engagement étatique a été axé vers la constitution de centres chorégraphiques nationaux (CCN), tournés initialement vers un soutien à la création de spectacles et peu en faveur de la diffusion, à la différence des centres dramatiques nationaux $(\mathrm{CDN})$.

Au cours des années 1980 et 1990, la concurrence s'est intensifiée et a pondéré la logique de

Daniel

Urrutiaguer

\author{
Maître de conférences HDR \\ IRET, Paris 3 \\ Sorbonne Paris Cité
}

service public. Plusieurs facteurs expliquent ce phénomène : la forte croissance démographique des entreprises chorégraphiques, à l'instar des autres genres artistiques ${ }^{1}$, un système de distribution des subventions basé sur l'appréciation par des experts professionnels de la valeur esthétique des spectacles créés, la concentration de la reconnaissance de la valeur axée sur la personne des porteurs de projets, ainsi que la montée en charge du régime de l'assurance-chômage des intermittents en complément d'une flexibilité nettement accrue des relations d'emplois's ${ }^{2}$ À partir des années 2000, la diffusion des spectacles ${ }^{3}$ et le développement des publics de la danse sont apparus peu à peu indispensables, et ce pour trois raisons : la marge disponible pour l'artistique des établissements culturels a été réduite, en raison d'une progression des subventions de fonctionnement inférieure à celle de leurs coûts fixes ${ }^{4}$, le spectacle vivant subventionné s'est vu appliquer une logique de rationalisation budgétaire dans un contexte de rigueur pour la gestion des comptes publics $^{5}$, enfin, les collectivités territoriales sont 
devenues très présentes dans le financement public des entreprises chorégraphiques.

L'objet de cet article est de dresser un tour d'horizon des connaissances statistiques sur la composition des publics de la danse et les motivations des spectateurs en analysant les enquêtes coordonnées par le ministère de la Culture ou des établissements culturels. Le faible nombre d'enquêtes réalisées ouvre un champ à des projections sur les capacités de développement du public en fonction des représentations mentales des protagonistes du monde de la danse contemporaine. Les divergences entre les aspirations des producteurs à l'allongement de leurs séries de représentations et les anticipations pragmatiques des diffuseurs sur les attentes de « leur » public sont la source de tensions récurrentes dans les processus de valorisation des œuvres chorégraphiques.

\section{Quelles connaissances sur les publics de la danse?}

La dernière enquête de grande ampleur sur les publics de la danse a été effectuée par JeanMichel Guy en 1988 auprès d'un échantillon de 10000 personnes représentatif de la population française âgée d'au moins 15 ans. La part des personnes ayant assisté à au moins un spectacle de danse professionnelle au cours des douze derniers mois était de $4 \%$ contre $5 \%$ pour un spectacle interprété par des amateurs ${ }^{6}$ et $7 \%$ pour les spectateurs de théâtre professionnel ${ }^{7}$. Les pourcentages étaient similaires pour les personnes ayant assisté à une représentation il y a plus d'un an et moins de quatre ans. Les inégalités sociodémographiques dans la fréquentation de la danse professionnelle sont marquées par une surreprésentation des cadres, puis des professions intermédiaires, des Parisiens, puis des Franciliens et des résidents des villes de plus de 100000 habitants, et des femmes. Les élèves, les étudiants, les agriculteurs et les ouvriers, les habitants des communes de moins de 100000 habitants sont plus présents dans le public des spectacles interprétés par des amateurs.

L'interrogation sur les motivations des spectateurs, rarement formulée dans les enquêtes, indique une prépondérance des raisons extrinsèques aux éléments du spectacle, notamment en termes de sociabilité, sur les raisons intrinsèques liées à la connaissance de composantes du spectacle. Cependant, les personnes interrogées étaient amenées à choisir trois raisons principales sur une liste déséquilibrée, composée de huit items extrinsèques contre cinq énoncés intrinsèques. Les liens entre les deux types de causes ne sont pas vraiment explorés dans le traitement des données? Les représentations sous-jacentes à la construction des catégories de danse par les professionnels et les spectateurs sont figurées par trois types de repérage pour classer le(s) dernier(s) spectacle(s) $\mathrm{vu(s),} \mathrm{l'un} \mathrm{par} \mathrm{l'enquêteur} \mathrm{à} \mathrm{partir} \mathrm{de} \mathrm{la} \mathrm{descrip-}$ tion effectuée par le spectateur interrogé, un autre par une question ouverte sur le type de genre vu, suivie d'une question à choix multiple avec cinq genres proposés (classique, moderne, folklorique, contemporain et jazz). Le genre contemporain est le mieux approprié par ses spectateurs, qui confirment leur classement opéré lors de la question ouverte au moment de la question fermée, à l'inverse d'une part importante des publics des autres genres, qui ne se prononcent pas sur l'un des cinq choix proposés par l'enquêteur ${ }^{10}$. 
Cet indice pourrait refléter l'existence d'une communauté d'interprétation si les échelles de jugement étaient similaires ${ }^{11}$. Il s'agit plutôt d'un effet suscité par une culture chorégraphique plus pointue propre aux amateurs éclairés de danse contemporaine. Comme l'indique une analyse par correspondances multiples pratiquée sur un sous-échantillon de 600 personnes, ce sont les spectateurs les plus motivés par des raisons intrinsèques qui cumulent le plus la fréquentation des musées, des expositions, des salles de cinémas, des autres spectacles vivants, et pour lesquels les artistes et les enseignants sont les plus présents ${ }^{12}$.

La comparaison des enquêtes nationales du ministère de la Culture et de la Communication de 1997 et $2008^{13}$ indique une stabilité de la part des Français ayant vu un spectacle de « danse professionnelle » au cours des douze derniers mois ( $8 \%)$, une régression de cette part pour les spectacles de « danses folkloriques » (de $13 \%$ à $10 \%$ ), alors que la proportion a progressé pour le théâtre (16\% en 1997, $19 \%$ en 2008). Les différenciations selon le sexe et la nomenclature des catégories socioprofessionnelles sont restées similaires, tandis que la surreprésentation des diplômés du supérieur, des résidents des villes de plus de 100000 habitants, des Parisiens intramuros s'est accentuée. Contrairement au théâtre, aux concerts, à l'opéra et aux danses folkloriques, les publics de la « danse » se sont rajeunis, la part des personnes ayant vu au moins un spectacle de danse au cours des douze derniers mois étant passée de $11 \%$ à $15 \%$ pour les $15-19$ ans et de $7 \%$ à $13 \%$ pour les $20-24$ ans.

Les enquêtes menées par des établissements culturels ne sont pas légion et sont conduites en fonction des données disponibles sur les logiciels de billetterie. Le lieu de résidence est un critère habituellement bien renseigné, ce qui permet de mesurer l'étendue de la zone de chalandise et de pratiquer, de façon plus ou moins avouée, un géomarketing en concentrant les efforts de communication sur les zones les plus densément peuplées en spectateurs de la structure. L'objectif est aussi de mesurer la part du public local, un critère auquel les collectivités territoriales sont particulièrement sensibles pour allouer leurs subventions. Ainsi, les enquêtes sur le public du Centre national de la danse (CND) réalisées depuis 2005 mesurent la répartition de l'origine géographique des spectateurs, des inscrits à la médiathèque, des clients des publications, des participants à l'entraînement régulier du danseur et du public des lundis de la santé ${ }^{14}$. Au cours de la saison 2011-2012, les Parisiens ont représenté presque la moitié des spectateurs de danse contre presque le quart pour les habitants de Seine-SaintDenis. Du fait de leur proximité géographique mais aussi de leur composition sociodémographique avec un nombre plus important d'artistes et d'enseignants, les $\mathrm{X}^{\mathrm{e}}, \mathrm{XI}^{\mathrm{e}}, \mathrm{XVIII}^{\mathrm{e}}, \mathrm{XIX}^{\mathrm{e}}$ et $\mathrm{XX}^{\mathrm{e}}$ arrondissements ont représenté presque les deux tiers des spectateurs parisiens, tandis que les Pantinois ont constitué environ $35 \%$ des spectateurs séquanodionysiens. Le poids de la Seine-Saint-Denis est logiquement moins prégnant pour les rencontres professionnelles ${ }^{15}$.

\section{Les projections sur les publics dans la programmation de la danse par les établissements culturels}

Les choix de programmation participent au développement d'un micromarché de la diffusion, 
spécifique à chaque établissement culturel. Le processus de décision peut reposer sur une relation de confiance avec un chorégraphe dont les projets sont accompagnés sur la durée pour la maturation de sa trajectoire artistique. En dehors de ces relations de fidélité, et en l'absence d'études poussées sur le public, les directions des établissements culturels sont amenées à anticiper le degré de succès des spectacles. Leurs projections se fondent sur des contacts avec des habitués du lieu et la construction imaginée des perceptions des spectateurs «moyens" selon la notoriété des chorégraphes et l'originalité esthétique des spectacles. La péréquation des risques est en effet nécessaire dans la construction d'une programmation afin d'assurer sa viabilité économique, tout en laissant une place à des propositions innovantes et à des compagnies peu renommées.

La programmation des spectacles de danse professionnelle a progressé dans les années 1990. Leur part dans les représentations des scènes nationales est passée de 10,7 \% en 1991-1992 à $13,6 \%$ en 1998-1999, tandis que celle de la danse dans les entrées payantes a progressé de $12,3 \%$ à $18,3 \%$ sur cette période, ce qui traduit une hausse du public de la danse plus rapide que l'offre. Le poids de la danse contemporaine dans le total des représentations de danse est estimé à $75 \%$ en 2007-2008 sur un échantillon de 29 scènes nationales. Celles-ci ont plus privilégié les compagnies que les $\mathrm{CCN}^{16}$. La part des spectacles de danse dans le total des représentations du Théâtre de la Ville est passée en moyenne de $20,1 \%$ dans les années 1970 à 57,1 \% entre les saisons 1996-1997 et 2007-2008. Pour les deux structures non franciliennes dominantes, le nombre de représentations chorégraphiques a augmenté entre 1986 et 2005 de $68 \%$ pour le Festival Montpellier Danse et de $83 \%$ pour la Maison de la Danse de $\operatorname{Lyon}^{17}$, la part moyenne de la danse contemporaine dans le total des représentations ayant été respectivement de $75 \%$ et de $50,6 \%{ }^{18}$.

Dans le cas du Théâtre de la Ville, le nombre de passages par compagnie entre 1969 et 2008 est corrélé positivement avec les productions des CCN. Alors que 40,6\% des compagnies n'ont été programmées qu'une seule fois, cela n'a été le cas que pour $15,8 \%$ des CCN, alors que $63,2 \%$ des CCN l'ont été au moins six fois contre une moyenne de $19,4 \%$ pour l'ensemble des compagnies ${ }^{19}$. Le Théâtre de la Ville a joué un rôle de tremplin pour certains chorégraphes, qui ont augmenté leur réputation corporative et constituent ainsi, après leur consécration institutionnelle, une force d'attraction auprès des spectateurs abonnés. Les scènes nationales programment davantage des compagnies qui ne dirigent pas un $\mathrm{CCN}$, dans la mesure où elles disposent d'un budget plus limité et qu'elles sont soutenues par l'Office national de la diffusion artistique (ONDA), organisme qui aide à la diffusion de spectacles risqués en raison de leur caractère innovant et de la faible notoriété du chorégraphe. La garantie financière de l'ONDA correspond à la prise en charge du déficit d'exploitation des représentations dans une proportion variant du quart au huitième de ce dernier selon une relation inversement proportionnelle avec le budget de l'établissement. Environ le quart de ces aides a concerné le secteur de la danse entre 2009 et $2012^{20}$. Cependant, la tentative de bonification de cette garantie pour une seconde représentation du même spectacle de danse, en assumant $20 \%$ du coût du contrat de cession, s'est heurtée à 
un échec. Les programmateurs n'avaient pas le sentiment de pouvoir accroître le public par une extension de l'offre, et les demandes d'aides spontanées ont été centrées sur des spectacles de chorégraphes consacrés tels Philippe Decouflé ou José Montalvo ${ }^{21}$.

Plusieurs indices indiquent un renversement récent dans les anticipations des établissements culturels sur le développement des publics de la danse. ARCADI (agence culturelle d'île-deFrance) a conduit une enquête sur la diffusion de la danse en Île-de-France entre 2003 et 2012. Cette dernière met en évidence, après une période d'expansion, un recul du nombre de lieux programmant des spectacles de danse de $26 \%$ entre 2008 et 2012, du nombre de représentations chorégraphiques de $18 \%$ entre 2007 et 2012 (à l'exception d'un point haut au cours de la saison 2010-2011), ainsi qu'une régression de la diversité des pièces programmées à partir de 2008, notamment pour le nombre de chorégraphes diffusés ${ }^{22}$. De façon contrastée, la progression de la programmation chorégraphique s'est poursuivie pour les treize «lieux de la danse $»^{23}$, qui sont des établissements subventionnés ayant diffusé le quart des représentations franciliennes de danse entre 2003 et 2012, et pour les lieux à label national en dehors de deux leaders ${ }^{24}$, le CND, créé en 1998, et le Théâtre national de Chaillot, plus orienté vers un accueil de la danse à partir de 2008. La baisse a été continue depuis 2007-2008 pour les théâtres de ville, les friches et lieux gérés par des compagnies et les lieux non dédiés au spectacle vivant ${ }^{25}$. La couverture de la garantie financière de Réseau en Scène Languedoc-Roussillon, construite sur le modèle de l'ONDA, a connu également un recul entre les périodes
2008-2010 et 2011-2013 pour le nombre d'œuvres (52 contre 39) et le nombre de représentations (105 contre 88), tandis que le nombre d'établissements bénéficiaires a un peu augmenté (32 contre 35) ${ }^{26}$. Dans un contexte de baisse de la marge disponible pour l'artistique des établissements culturels, la programmation en danse est considérée comme plus risquée et est concurrencée notamment par les arts du cirque et de la rue.

\section{Les aspirations des compagnies chorégra- phiques au développement des publics}

L'enquête sur les territoires et les ressources des compagnies en France, que j'ai coordonnée avec Philippe Henry ${ }^{27}$, confirme la plus faible diffusion des spectacles de danse, déjà mise en évidence par celle d'ARCADI en 200728. Dans l'échantillon quantitatif, constitué de 572 répondants dans les domaines du spectacle vivant non musical, le nombre annuel médian de représentations est estimé en 2009 à 11 pour la danse contre 28 pour le théâtre, et le budget annuel médian est respectivement de 61000 euros et 82865 euros. Les analyses en composantes principales des échantillons quantitatif et qualitatif (51 études de cas) révèlent que la typologie fondée sur le centre de gravité territorial de la diffusion des spectacles est la plus discriminante, au-delà des différences de genre artistique. Une corrélation positive existe entre le poids des représentations en dehors de la région du siège social, l'accès à la programmation des établissements culturels ayant un label national et la probabilité du conventionnement par la direction régionale des Affaires culturelles (DRAC) ${ }^{29}$. Les négociations entre les compagnies et les établissements culturels pour impliquer ces derniers dans la coproduction et 
la diffusion des spectacles sont plus ou moins tendues : tout dépend de la réputation corporative du chorégraphe, fondée en général sur sa capacité à rayonner à l'intérieur et à l'extérieur de l'Hexagone. L'enquête d'ARCADI de 2013 confirme le recoupement des circuits de diffusion avec la stratification des marchés-réseaux de production : Sombrero de Philippe Decouflé a été joué dans les «trois phares » (Théâtre de la Ville, CND, Théâtre national de Chaillot), tandis que Traversée d'ombres de Fabrice Guillot et Geneviève Mazin, praticiens de la danse verticale, a été plus diffusé dans les théâtres de ville et les établissements non culturels, moins rémunérateurs ${ }^{30}$.

L'échantillon qualitatif de l'enquête que nous avons coordonnée avec Philippe Henry souligne un durcissement de ces relations. Il s'agit d'un élément récurrent dans les entretiens avec les chorégraphes et les administrateurs de compagnies. Leur croyance dans le développement des publics de la danse contemporaine, par un allongement des séries de représentations susceptible d'activer le bouche à oreille, se heurte au scepticisme des programmateurs. Les bilans d'activité, les comptes de résultat et les DADS (déclarations automatisées des données sociales) sur les salaires apportent un éclairage plus précis : pour l'ensemble des compagnies du spectacle vivant non musical, entre 2008 et 2009 , et en comparaison avec la période 2007-2008, le nombre des représentations diffusées a nettement augmenté, mais le nombre de représentations par spectacle a progressivement diminué. Les tensions se sont cristallisées autour d'une baisse des recettes par représentation de l'ordre de $40 \%$ entre 2007 et 2009, dont la moitié est liée à une réduction de la taille moyenne des équipes artistiques et techniques $^{31}$. Par ailleurs, la réduction de la masse salariale artistique et technique par représentation a été la plus forte pour les compagnies « excentrées », qui concentrent l'essentiel de leur diffusion dans les espaces national et international. Les cessions de représentations dans une zone proche du coût du plateau sont fréquentes ${ }^{32}$.

Cette opposition dans les projections sur le développement des publics de la danse contemporaine se manifeste aussi par des accusations sur le manque de culture chorégraphique des directions d'établissements culturels, souvent plus familiarisées avec le monde du théâtre. Le rapport commandité par la ministre de la Culture et de la Communication Catherine Trautmann, pour explorer la faisabilité de la création d'un « théâtre national de la danse à Paris », notait en 1999 «le déficit, si souvent souligné, de programmateurs connaissant vraiment la danse, son histoire, sa géographie comme ses nouvelles aventures » pour expliquer « la faiblesse du nombre de spectacles de danse» et « un formatage trop souvent stéréotypé des programmations $»^{33}$.

Les liens de fidélité des spectateurs avec un chorégraphe semblent plus accentués que pour un metteur en scène. Ils se manifestent par une envie de nomadisme plus forte afin de suivre les créations d'une compagnie dans les différents établissements culturels, notamment quand celleci est en résidence. On peut y voir un effet de la diffusion plus limitée des pièces chorégraphiques. Ainsi la part des Parisiens dans le public du Forum du Blanc-Mesnil, une scène conventionnée située à 12,5 kilomètres de Paris, était la plus forte pour les spectacles de danse avec une proportion d'environ $25 \%$ des spectateurs contre 
$16 \%$ pour le théâtre entre 2007 et 2009 d'après les données des fichiers de spectateurs.

Les directeurs d'établissements culturels sont logés à la même enseigne que les créateurs : ils doivent soigner leur réputation corporative. Celle-ci dépend de leur capacité à dénicher de nouveaux talents; ils sont donc à la recherche de nouveautés stylistiques dans un contexte d'incertitude radicale quant à la reconnaissance de la qualité ${ }^{34}$. Le risque de rejet d'un choix innovant par les pairs les contraint souvent au suivisme et à la formation de bulles spéculatives sur des artistes peu connus, qui concentrent à un moment donné l'attention des programmateurs mais peuvent être « lâchés » s'ils ne se renouvellent pas suffisamment. Ce phénomène est très prégnant dans le monde de la danse contemporaine d'après les études de cas de l'enquête publiée en 2011.

Une chorégraphe trentenaire, formée dans l'école d'un CCN, estime avoir bénéficié de cet effet lors de sa première création, fondée sur le croisement entre une danse théâtralisée et l'univers musical d'un compositeur rock. Son administratrice a constaté une fréquentation inattendue de nombreux programmateurs lors des premières présentations, comme si une rumeur s'était construite autour de son travail. Une chorégraphe quinquagénaire, qui crée des solos autour d'une recherche inspirée d'un concept d'architecture moléculaire, concrétisé par la mise en contact de sa peau avec des matières tubulaires, a profité, elle aussi, de cet effet lors de la présentation de sa première création. Par la suite, elle a observé une diminution de l'intérêt pour ses autres créations axées autour de la même thématique esthétique. Selon un autre chorégraphe quinquagénaire, les médias et les institutions favorisent davantage l'émergence et les cultures urbaines ${ }^{35}$.

\section{L'éducation chorégraphique et l'hétérogénéité de la demande}

Des efforts éducatifs sont nécessaires pour permettre d'appréhender puis d'apprécier des spectacles chorégraphiques qui souvent offrent des représentations originales et peu familières $\mathrm{du}$ corps dans l'espace. Par exemple, les amateurs de théâtre seront désarçonnés par l'absence de narration lisible, et les amateurs de danse « technique » seront hostiles à toute proposition impliquant une gestuelle minimaliste.

Les révoltes de spectateurs amateurs de la « danse qui danse » contre la « non-danse » sont devenues plus fréquentes et ciblées sur certains chorégraphes, notamment au Théâtre de la Ville. Le 7 février 2009, la représentation de la pièce Turba de Maguy Marin a suscité un chahut émanant d'une partie du public, désappointée et agacée par la théâtralité des lents déplacements et l'immobilité à l'œuvre lors de la lecture du De la nature des choses de Lucrèce. La montée sur le plateau d'un spectateur pour exécuter des entrechats a provoqué l'arrêt du spectacle par la chorégraphe, puis sa reprise après un appel à quitter la salle destiné à ceux qui ne supportaient pas ce travail - paradoxalement, peu sont sortis. David Mati, conseiller sectoriel en danse de la DRAC francilienne, a pu constater qu'une préparation en amont limitait les sources d'incompréhension : il a assisté à une représentation d'Umwelt, pièce créée toujours par Maguy Marin en 2004, dans une salle attentive et pleine à l'Espace Germinal de Fosses, où la programmation s'accompagnait 
d'actions de médiation, alors que les spectateurs du Théâtre de la Ville sortaient en masse ${ }^{36}$.

L'analyse par correspondances multiples des discours des spectateurs de danse sur leurs attentes par Guy en 1988 a fait apparaître que l'argumentation heuristique, sensible à la poétique de la danse notamment contemporaine, n'était partagée que par $13 \%$ des spectateurs. Une autre minorité, équivalente en proportion, légitimait le respect des traditions. Quarante pour cent des discours étaient de nature formaliste, avec une attention à la pureté des mouvements et à la beauté canonique des corps, tandis que $34 \%$ mettaient en avant la sensibilité à l'expression du caractère des danseurs, tant individuel que groupal ${ }^{37}$.

Les directions des établissements culturels ont compris que la danse contemporaine était perçue comme un art complexe. Cela les a conduites à demander aux compagnies programmées de s'engager dans des dispositifs d'action culturelle. Souvent, ainsi que le confie Claire Harsanyi, chargée de production, le nombre d'interventions pédagogiques est plus élevé pour une compagnie chorégraphique que pour une compagnie de cirque $^{38}$. Dans la mesure où l'on manque de connaissances sur les attentes du public, il s'agit de fournir des repères permettant de situer le travail chorégraphique. Les rencontres avec un artiste ou la conduite d'ateliers peuvent montrer qu'un savoir-faire technique et un engagement personnel dans l'exécution singulière de mouvements, même s'ils sont minimalistes, sont aussi indispensables. Le problème du sens est souvent un obstacle réel : l'enjeu consiste donc à inviter les spectateurs à ne pas chercher à comprendre, à leur faire comprendre que ce n'est pas premier et qu'ils doivent plutôt se laisser émouvoir par la gestuelle et la rythmique des danseurs. Et c'est là qu'un autre écueil surgit : certains, non convaincus par cette approche, peuvent se sentir accusés implicitement d'incompréhension et d'insensibilité, ce qui les démotive. En outre, les artistes possèdent des compétences pédagogiques variables. Leur implication dans l'action culturelle dépend des orientations du projet artistique des compagnies, du partage de temps proposé par l'établissement d'accueil avec les activités de création, ainsi que du degré de perception d'un enrichissement mutuel au contact des pratiques de non professionnels.

Dans l'exploitation de son enquête de 1988, Guy a minoré l'impact de la pratique de la danse en amateur sur la fréquentation des spectacles de danse professionnelle. Pourtant, la part des personnes ayant vu au moins un spectacle de danse au cours des quatre dernières années était de $24 \%$ pour les pratiquants actuels ou anciens de la danse, contre $11 \%$ pour ceux qui n'avaient jamais fait de danse. Cette part était encore plus élevée (39\%) quand était pris en compte un contact par un enfant pratiquant ${ }^{39}$. L'enquête de Donnat sur les amateurs en 1994 apporte des résultats plus nuancés sur la diversité des comportements selon le genre pratiqué. Les anciens amateurs de danse sont les plus nombreux à déclarer que la danse était le loisir qu'ils ont pratiqué le plus longtemps et qui les a le plus marqué. Cet avis est surtout partagé par les praticiens de la danse classique ou contemporaine. Ceux-ci sont aussi les plus nombreux à avoir assisté à un spectacle de danse professionnelle, devant les amateurs de danses folkloriques, tandis que les praticiens de danses de salon sont les moins ouverts aux spectacles 
professionnels ${ }^{40}$. Selon l'enquête «Pratiques culturelles et sportives » réalisée par l'INSEE en $2003,42 \%$ des praticiens amateurs vont voir des spectacles de danse, contre $11 \%$ pour les non-praticiens ${ }^{41}$; une enquête ministérielle datant de 2008 corrobore dans une moindre mesure ces chiffres : $28 \%$ pour les amateurs de danse contre une moyenne de $8 \%$ pour l'ensemble des français ${ }^{42}$.

Ces indicateurs montrent un lien, certes non généralisé, entre la pratique des amateurs et la fréquentation de la danse professionnelle. La minoration de cet impact peut s'inscrire dans un discours déniant l'efficacité des ateliers de pratique artistique en comparant leur coût avec les retombées quantitatives en termes de public.

\section{Conclusion}

Dans un contexte où le pouvoir de marché des établissements culturels s'est renforcé dans la chaîne de valeur du spectacle vivant, le monde de la danse contemporaine se caractérise par une vitalité de la production chorégraphique et une faible étude des différentes motivations des spectateurs et des praticiens amateurs. La logique du service public chorégraphique se trouve confrontée à celle de la rationalisation budgétaire ; cette contrainte d'équilibre budgétaire, en situation d'incertitude sur la réception de la qualité artistique, incite de nombreux programmateurs à privilégier dans leurs choix la notoriété rassurante des chorégraphes les plus en vue. La nécessité d'innovations esthétiques pour capter l'attention des prescripteurs de jugement renforce les spéculations sur les gains en réputation corporative de compagnies encore peu connues. Enfin, les représentations imaginaires des publics de la danse alimentent les défauts de coordination entre producteurs, diffuseurs, collectivités publiques et spectateurs.

\section{Remerciements}

Je remercie Cyril Duchêne (Centre national de la danse) pour les échanges autour des questions abordées. 
$\mathrm{N} \cdot \mathrm{O} \cdot \mathrm{T} \cdot \mathrm{E} \cdot \mathrm{S}$

1. Entre 2000 et 2008 , le nombre d'employeurs du spectacle vivant a augmenté de $47 \%$ pour la branche professionnelle du spectacle vivant et de $95 \%$ pour les employeurs occasionnels des autres branches (Observatoire prospectif du spectacle vivant, Tableau de bord de l'emploi et de la formation dans le spectacle vivant, Paris, Ministère de la Culture et de la Communication, 2011 : 11).

2. Selon les données de la Caisse des congés du spectacle, la durée moyenne des contrats de travail des intermittents du spectacle est passée de 19 jours en 1989 à 6 jours en 2000, puis 4 en 2007 (calculs personnels à partir de CNPS, Emploi et Spectacle. Rapport de la commission permanente sur l'emploi $d u$ CNPS. 2010-2011, Paris, CNPS, 2011, p. 18), dont environ 2 pour les artistes et 6 pour les techniciens. Ce raccourcissement s'accompagne du maintien d'un employeur principal qui assurait en moyenne $65 \%$ des engagements des artistes interprètes en 2001 selon les calculs de P.-M. Menger et C. Marchika (Comptes et comportements dans l'assurance-chômage des intermittents, Paris, CESTA, 2004).

3. Par exemple, la composition de la commission consultative de l'aide à la création chorégraphique de la DRAC en Île-de-France s'est modifiée en ce sens entre 2009 et 2013. La part des représentants des directions d'établissements est passée de 41,2 \% à 52,6\% au détriment de celle des critiques chorégraphiques, réduite de $17,6 \%$ à 5,3\%. Une «personne compétente issue du public » siège dans cette commission depuis 2010. Le nombre d'observateurs des collectivités territoriales est passé de 5 à 8 au cours de cette période. Alors que l'ONDA et Cultures France étaient les seuls autres organismes invités à suivre les travaux en 2009, le CND et Arcadi l'ont été respectivement depuis 2010 et 2011 .

4. Le rapport sur le financement du spectacle vivant, commandité par le ministre de la Culture Frédéric Mitterrand en 2012, indique que le pouvoir d'achat des subventions reçues par les quinze $\mathrm{CDN}$ et scènes nationales franciliens a perdu 6 millions d'euros en sept ans du fait de l'absence d'indexation des aides publiques sur les prix et du retrait de certaines collectivités territoriales (S. Dorny et al., Financement du spectacle vivant. Développer, structurer, pérenniser, Paris, Ministère de la Culture et de la Communication, 2012, p. 29).

5. Alors que le budget du ministère chargé de la Culture à prix constants a été multiplié par 6,5 de 1960 à 1985 , il a augmenté de $33 \%$ de 1990 à 2000 , puis de 8,5\% de 2000 à 2010 (J.-F. Chougnet, « L'effort public pour la culture », in P. Poirrier (dir.), Politiques et pratiques de la culture, Paris, La Documentation française, 2010, p. 39).

6. J.-M. Guy, Les Publics de la danse, Paris, La Documentation française, 1991, p. 60.

7. J.-M. Guy et L. Mironer, Les Publics du théâtre, Paris, La Documentation française, 1988, p. 21.

8. J.-M. Guy, op. cit., p. 64.

9. Ibid. , pp. 77-79.

10. Ibid., pp. 90-99.

11. Le sociologue J.-P. Esquénazi (Sociologie des œuvres. De la production à l'interprétation, Paris, Armand Colin, 2007, pp. 76-88) se réfère à ce concept, qui a été proposé initialement par le professeur de littérature S. Fish ("Interpreting the "Variorum" », Critical Enquiry, vol. 2, n 3, 1976, pp. 465-485) pour mettre en avant l'impact de l'expérience antérieure du lecteur sur le sens donné à un texte. En s'inspirant de la démarche de l'historien M. Baxandall, il s'agit de caractériser le processus d'interprétation à la fois par une description de l'œuvre et par un jugement de valeur. 
12. J.-M. Guy, op. cit. , pp. 191-197.

13. O. Donnat, Les Pratiques culturelles des Français. Enquête 1997, Paris, La Documentation française, 1998 ; O. Donnat, Les Pratiques culturelles des Français à l'ère numérique. Enquête 2008, Paris, La Découverte, 2009.

14. Il s'agit de rencontres professionnelles autour de conférences sur les risques de santé impliqués par le métier de danseur.

15. CND, Origine géographique des publics de la saison 11>12, Pantin, CND, p. 9.

16. P. Germain-Thomas, Politique et marché de la danse contemporaine en France (1975-2009), thèse de doctorat sous la direction de P. Urfalino, Paris, EHESS, 2010, pp. 280-281.

17. Guy Darmet, le directeur artistique de cette structure ouverte en 1980, a également créé la Biennale de Lyon en 1984. Il a été remplacé par Dominique Hervieu en juillet 2011.

18. P. Germain-Thomas, op. cit. , pp. 269 et 272.

19. Ibid., p. 260.

20. ONDA, Bilan d'activité 2012, Paris, ONDA, 2013, p. 13.

21. Entretien de D. Urrutiaguer avec S. Dondi, ancienne conseillère en danse de l'ONDA et présidente du Réseau en Scène Languedoc-Roussillon, réalisé le 26 juin 2013.

22. ARCADI, Cultures en Île-de-France \#2, février 2013, pp. 8, 9 et 29.

23. À Paris, le Centre Pompidou, L'Étoile du Nord, La Ménagerie de Verre, le Parc de la Villette, Le Regard du Cygne, le Théâtre des Abbesses, le Théâtre de la Bastille, le Théâtre de la Cité internationale, le Théâtre Dunois, le Théâtre du Lierre jusqu'en 2010, et dans la proche couronne la Maison des arts de Créteil, le Théâtre Jean-Vilar à Suresnes et le Théâtre de Vanves. 24. Ils sont classés parmi les « trois phares » avec le Théâtre de la Ville.
25. ARCADI, op. cit., p. 13.

26. Source : S. Dondi, courriel adressé à D. Urrutiaguer le 4 juillet 2013.

27. D. Urrutiaguer et P. Henry, Territoires et ressources des compagnies en France, Paris, Ministère de la Culture et de la Communication, 2011.

28. ARCADI, Consultation des compagnies chorégraphiques d'île-de-France, rapport final, 2007. 29. D. Urrutiaguer, P. Henry et C. Duchêne, «Territoires et ressources des compagnies en France », Cultures Études n ${ }^{\circ} 2012-1,2012$, pp. 14, 15.

30. ARCADI, op. cit., 2013, p. 31.

31. D. Urrutiaguer et al., op. cit, 2012, p. 7.

32. P. Germain-Thomas, La Danse contemporaine, une révolution réussie?, Toulouse, L'Attribut, 2012, p. 102.

33. A. Chiffert et al., Pour un théâtre national de la danse à Paris, Paris, Ministère de la Culture et de la Communication, 1999, p. 3.

34. L. Karpik, L'Économie des singularités, Paris, 2007.

35. D. Urrutiaguer et P. Henry, op. cit., p. 126.

36. Entretien de D. Urrutiaguer avec D. Mati réalisé le 28 juin 2013.

37. J.-M. Guy, op. cit., p. 360.

38. C. Harsanyi, «Une réalité vécue », 2005. Journées de réflexion, Paris, Micadanses, p. 30.

39. J.-M. Guy, op. cit., pp. 67 et 68.

40. O. Donnat, Les Amateurs. Enquête sur les activités artistiques des Français, Paris, La Documentation Française, 1996, pp. 199, 217 et 218.

41. Effectuée dans le cadre des «Enquêtes permanentes sur les conditions de vie » (EPCN), in DMDTS, « Les publics du spectacle vivant », Repères $n^{\circ} 4,2008$, p. 10. 42. L. Babé, «Les publics de la danse », Repères DGCA $n^{\circ} 6.03,2012$, p. 12. 


\section{$R \cdot E ́ \cdot S \cdot U \cdot M \cdot E$}

Peu nombreuses, les enquêtes sur les spectateurs de la danse contemporaine sont centrées sur des variables sociodémographiques à l'échelle nationale ou sur le lieu de résidence pour les établissements culturels. Néanmoins, cette faible connaissance des différentes motivations des spectateurs et des praticiens amateurs révèle des oppositions entre les lieux de spectacle et les compagnies dans les représentations des goûts esthétiques. L'accès aux marchés-réseaux de production est stratifié selon la réputation corporative des producteurs, qui fait l'objet de spéculations sur la promotion de « nouveaux talents » par les établissements culturels. Le cycle de diffusion moyen des spectacles est court et dépend de la notoriété des chorégraphes. La part de la danse dans la programmation des lieux non spécialisés a reculé, car ces derniers sont tenus à augmenter leurs revenus propres. De plus, les craintes des directions de lieux sur la complexité cognitive des chorégraphies alourdissent leur demande d'action culturelle aux compagnies.

\begin{abstract}
Audience representations in the world of contemporary dance

Rare are the studies on contemporary dance audiences, and they focus on the socio-demographic variables on a national scale or on the place of residence for the cultural establishments. Nevertheless, this poor knowledge of the different motivations of audiences and amateur dancers reveals oppositions between the performance venues and the companies in the representations of aesthetic taste. The access to product market networks is stratified according to producers' corporate reputation, which is the subject of speculations regarding the promotion of "new talents" by
\end{abstract}

cultural establishments. The average distribution cycle of performances is short and depends on the choreographers' celebrity. The percentage that the dance has in the programming of non-specialized venues has slipped, as the latter have held to increasing their own income. In addition, venue managers' fears over the cognitive complexity of choreographies weighs on their request to companies for cultural action. 